\title{
The natural appearance of unnatural incline speed
}

\author{
DOUG ROHRER \\ University of South Florida, Tampa, Florida
}

\begin{abstract}
In three experiments, college students provided judgments about a marble's speed along a nonlinear incline. Each experiment revealed widespread support for the slope-speed belief, a mistaken belief holding that an object's speed at any point depends on the slope at that point. In truth, an object's incline speed varies with its elevation. In Experiment 1, participants relied solely on a diagram. In Experiments 2 and 3, participants observed computer animations depicting the descent of a marble at speeds conforming to either the slope-speed belief or Newtonian theory, and they rated the slopespeed version as more "natural" than the correctversion. The task in Experiment 1 gauged participants' consciously available knowledge, but the perceptual realism of the slope-speed animations suggests that the slope-speed belief is also held outside awareness. By contrast, virtually all previously identified false beliefs about motion appear unnatural once animated.
\end{abstract}

Numerous studies have revealed that people possess striking false beliefs about the qualitative motion of an object as it falls to the ground or rolls along an incline, even though these beliefs are at odds with common experiences. But the evidence for these beliefs is typically drawn from tasks in which participants base their judgments on a diagram or other static stimulus, like those in Figures 1A and $1 \mathrm{~B}$. In the bomb drop task of Figure 1B, for example, many people falsely predict that the bomb will fall straight down after it is dropped from a horizontally moving plane (McCloskey, Washburn, \& Felch, 1983).

Many of these beliefs prove unpopular once people have the opportunity to observe a computer animation that conforms to the belief. In one such study of the bomb drop task, for instance, observers rated the impossible straightdown trajectory as less natural than the correct Newtonian trajectory (Kaiser, Proffitt, Whelan, \& Hecht, 1992). Hence, although a straight-down trajectory may seem natural to those relying solely on the diagram in Figure 1B, it does not appear realistic to those looking at an animated version. Several authors have noted that this dissociation illustrates a distinction between people's consciously available information about motion, or explicit knowledge, and their unconscious implicit knowledge (e.g., Hecht \& Bertamini, 2000; Hubbard, 1998; Kozhevnikov \& Hegarty, 2001). Hence, whereas some tasks may rely on people's explicit knowledge about motion, other tasks may tap their implicit knowledge.

The present experiments concern people's qualitative understanding of an object's incline speed along a nonlinear incline, as can be illustrated by the motion of roller coasters, skis, sleds, bicycles, toy cars, and so forth. The experiments specifically address a false belief known as

Correspondence should be addressed to D. Rohrer, Department of Psychology,PCD4118G, University of South Florida, Tampa, FL 33620 (e-mail: drohrer@chuma1.cas.usf.edu). the slope-speed belief, which was shown to be popular in a previous study gauging people's explicit knowledge (Rohrer, 2002). The present experiments used both an explicit task (Experiment 1) and two implicit tasks (Experiments 2 and 3), with the latter designed to measure whether the slope-speed belief is a part of people's implicit knowledge. Further details of the slope-speed belief are given after the following review of the literature regarding animation studies of false beliefs.

\section{False Beliefs That Appear Unrealistic Once Animated}

One commonly investigated false belief is illustrated by the C-tube task in Figure 1A. The marble is propelled through the tube lying flat on a horizontal surface, and participants predict the shape of the marble's path after it exits the tube. By Newton's second law, the marble rolls straight in the absence of nonzero net force. Yet some people predict that the marble will roll along a curved path after it leaves the tube (Catrambone, Jones, Jonides, \& Seifert, 1995; Kaiser, Jonides, \& Alexander, 1986; Kaiser, McCloskey, \& Proffitt, 1986; McCloskey, Caramazza, \& Green, 1980; McCloskey \& Kohl, 1983). To assess the influence of animation on this task, Kaiser, Proffitt, and Anderson (1985) presented either diagrams or animations illustrating the various choices; animation greatly benefited performance.

Similarly, Kaiser et al. (1992) showed that animation provided the insight necessary for solving other classic problems. One of these studies (McCloskey et al., 1983) concerned the bomb drop task shown in Figure 1B and described in the beginning of the introduction. Because the bomb has horizontal speed when it is released, it continues to move forward as it falls. McCloskey et al. found that many adults predict instead that the bomb will fall directly downward. However, Kaiser et al. (1992) found that the Newtonian animation was preferred over the "straight-down" animation. These authors also examined the influence of 


\section{A C-Tube}

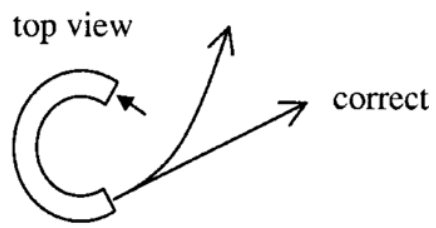

B Bomb Drop

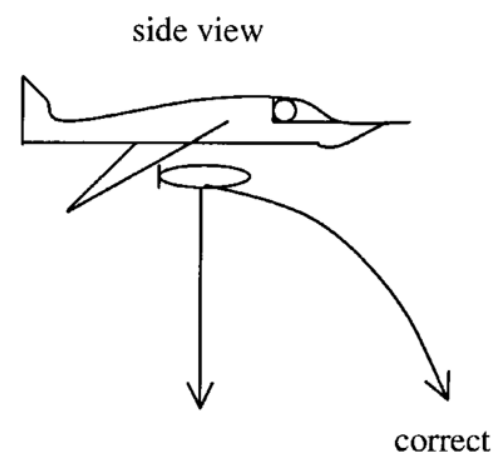

C Broken Pendulum

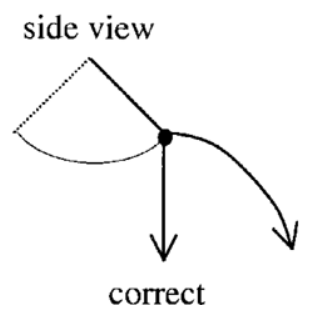

Figure 1. Previously reported false beliefs regarding position. (A) C-tube task. A marble is shot through the tube. (B) Bomb drop task. A horizontally flying plane drops a bomb. (C) Broken pendulum task. The string breaks just as the swinging bob reaches its highest point.

animation on the broken pendulum problem, illustrated in Figure 1C. Here, the suspended bob swings back and forth until the string breaks at the instant the bob reaches the highest point along its arc. Because the bob has no horizontal speed at this point, the bob falls directly downward. Yet many adults choose the nonvertical trajectory shown in Figure 1C (see, e.g., Caramazza, McCloskey, \& Green, 1981). When these options were animated in Kaiser et al.'s (1992) study, however, observers rated the Newtonian animation as the more natural of the two possibilities.

\section{Perceptually Realistic False Beliefs}

The animated versions of a few false beliefs appear natural. In Hecht and Bertamini (2000), participants considered the speed of a ball thrown by one person to another, with the ball first gaining and then losing altitude during its trajectory. In the explicit knowledge task, participants saw a diagram of this parabolic trajectory and selected the point of greatest speed. Many of them mistakenly predicted that the ball would reach its maximum speed at a point well beyond its release from the thrower's hand when, in fact, the ball begins to slow immediately upon release as it gains altitude. In a second experiment employing an implicit task, animations depicting this "magical post-release acceleration" were rated as more natural than the Newtonian animations for certain angles of release. The authors thus concluded that this belief is a part of both "explicit and implicit understanding" (p. 730).

In another study of trajectory motion, Kozhevnikov and Hegarty (2001) described an error regarding the effect of mass on an object thrown directly upward. In a study gauging explicit knowledge, participants predicted whether a heavy ball or a light ball would first reach a given heightthat is, which ball moves faster when thrown upward with equal force. In truth, the heavier ball reaches any given height before the lighter ball, because lighter objects are more susceptible to the impeding effects of air resistance. (This is why a thrown feather rapidly loses speed.) Yet most participants predicted that the lighter object was faster. In a second study designed to measure implicit knowledge, participants observed animations of an "upwardly thrown" square that was either large or small and thus purportedly perceived as "heavy" or "light," respectively. Just before the square reached its maximum height, it disappeared and then reappeared $150 \mathrm{msec}$ later as a stationary square. Observers then judged whether the square was located where it had disappeared. On average, the "remembered position" was too high, as if the mental representation of the square continued upward during its absence from the screen. This error is known as "representational momentum" (e.g., Hubbard, 1995). Notably, the size of the error was greater for the "light" square than for the "heavy" square, in keeping with the belief that lighter objects move faster than heavier objects during upward motion. Thus, Kozhevnikov and Hegarty concluded that this false belief was a part of these participants' implicit understanding.

\section{Incline Speed}

An object's incline speed at any point is unaffected by the slope at that point, and this is true regardless of the ramp shape. Formally, an object's incline speed $(v)$ is given by

$$
v=(2 g y)^{1 / 2},
$$

where $y$ denotes the object's net vertical drop since its release, $g$ equals its rate of acceleration near the surface of the earth, and $k$ depends on the object's shape and its distribution of mass (e.g., Halliday \& Resnick, 1981). The value of $k$ equals $10 / 7$ for uniformly dense spheres like the marbles considered in the present experiments, although the value of this parameter has no qualitative effect on an 
object's incline speed. By substituting these values for $k$ and $g$ in Equation 1, the incline speed of a marble is given by

$$
v=3.74 y^{1 / 2} \text {. }
$$

Notably, incline speed $(v)$ simply varies in direct proportion to the square root of its net vertical drop $(y)$, and this is true for any object that rolls or slides if the impeding effects of friction are negligible. This elegant relation is also true for free fall; Galileo discovered it by carefully timing the descent of a rolling sphere along linear inclines (Crawford, 1996; Drake, 1989). For the present study, though, only one implication of Equation 2 is relevant: As a marble rolls along an incline of any shape, its speed at any point depends on its net vertical drop at the point.

The presence of friction can complicate matters, because friction impedes speed. This complexity cannot be ignored, of course, because participants' views are shaped by experiences that are sometimes fraught with friction. Still, it is worth noting that friction often has very little effect on rolling speed. For instance, once a roller coaster is released from the top of the first hill, it can ascend an immediately subsequent hill that is virtually as high as the first. Nevertheless, participants may assume the presence of friction, and this possibility must be considered.

\section{Duration of a Descent}

Although the present experiments concern an object's incline speed, it is important to clarify the relation between an object's speed at a given point and the duration of its descent from the start to that point. As an illustration, Figure 2 includes two ramps that share a starting point and a finish point. Thus, the two ramps yield the same net vertical drop, and, consequently, both ramps produce the same speed at the finish (Equation 2). However, the two ramps produce different descent durations. In fact, the curved ramp yields a much quicker descent, even though the linear ramp has the shorter distance. In effect, the greater initial steepness of the curved ramp provides rapid acceleration, and the concomitant speed more than compensates for the longer rolling distance. In other terms, the initial steepness of the curve ramp allows the

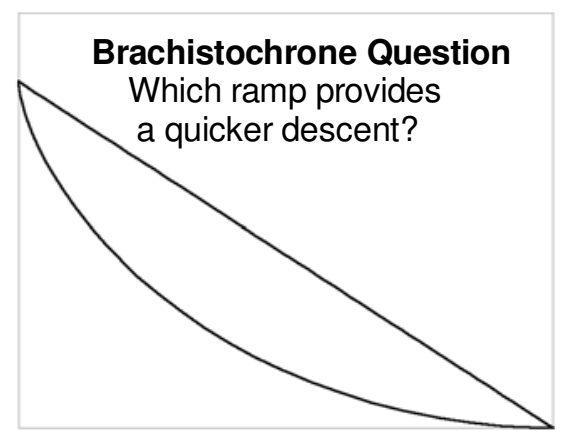

Figure 2. The brachistochrone problem. In a race between identical marbles, the curved ramp is quicker. Yet both shapes produce identical speeds at the finish, as each ramp generates the same net vertical drop. marble to quickly descend to points of lower elevation where speed is greater. In fact, the shape of the curve in Figure 2 is known as the curve of the brachistochrone ("shortest time"), because it is optimally quick (e.g., Anton, 1980; Rohrer, 1994, 2002).

A simpler task concerning descent duration is the race question. As shown in Figures $3 \mathrm{~A}$ and $3 \mathrm{~B}$, this is a race between two marbles along a "dip ramp" and a "hill ramp." These ramp shapes are mathematically specified in Appendix A. The two ramps are equal in length and share a start point and a finish point. Yet the dip ramp is much quicker, because the points within the "dip" lie at a greater net vertical drop than the points within the "hill." Hence, speed at every point within the "dip" is faster than speed at every point within the "hill."

The race question is difficult. In a study reported by Rohrer (2002), more than half of a college student sample incorrectly predicted a tie after viewing a three-dimensional model of the ramps (but not observing any motion). In the written justifications for this false prediction, many explained erroneously that both the dip and the hill include one "fast" downhill and one "slow" uphill and therefore "cancel each other out." Hence, although speed actually depends on vertical drop, these students seemingly attributed incline speed to slope. These responses suggested the prevalence of a false belief that serves as the focus of the present study.

\section{The Slope-Speed Belief}

The slope-speed belief holds that an object's incline speed at any point depends on the slope at the point. Two instantiations are specified by this belief. First, an object's incline speed is greater at a "downhill point" than at an "uphill point." Second, an object's incline speed is greater at a steep "downhill point" than at a gradual "downhill point." (The terms "downhill point" and "uphill point" are used to describe a point on a downhill or uphill, respectively, although, technically, a point does not have slope.)

The slope-speed belief is violated by real-world experiences. A roller coaster's speed near the top of the first downhill is slower than its speed near the bottom of the following uphill, because speed depends on net vertical drop. The same is true for a skateboarder who traverses a U-shaped ramp, as speeds are greatest along the flat portion at the bottom. Other examples include the incline speed of skiers, nonpedaling cyclists, and drivers of toy cars.

Nevertheless, the slope-speed belief proved popular in a study by Rohrer (2002) that included the speed question shown in Figure 3C. This scenario is not a race. Instead, participants must predict which marble, if either, rolls faster at the indicated critical point while observing a three-dimensional model of each ramp (without having the opportunity to observe any motion). Because an object's speed depends on its net vertical drop, incline speed is greater at the "uphill point" than at the "downhill point" (by Equation 2). Yet most of the participants incorrectly predicted greater speed at the "downhill point," consistent with the slope-speed belief. Of these participants, virtually all explained their answer by citing the difference in slope. That these participants attributed their responses to 
A Hill Ramp Specifications

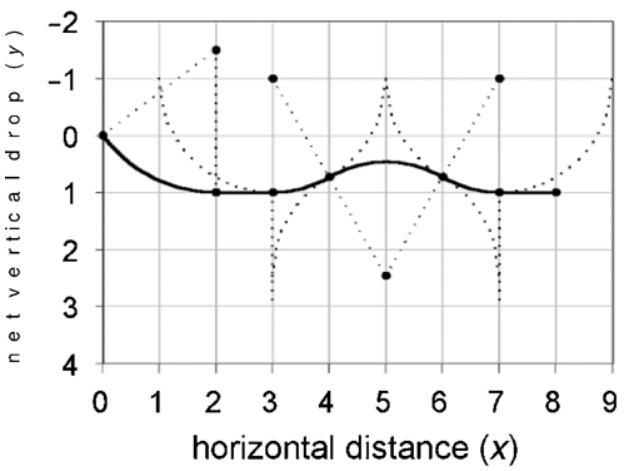

B Race Question (brief version)

The marbles are identical.

The marbles are released simultaneously.

Which marble wins, if any?

* Marble A (along the dip ramp)

* Marble B (along the hill ramp)

* Neither-the marbles tie

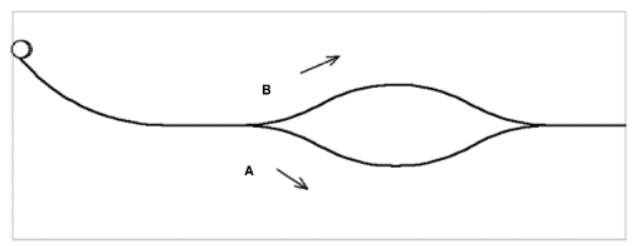

Answer Dip ramp is quicker, because all points along dip lie "below" all points along hill

Results Most predicted a tie

\section{Dip Ramp Specifications}

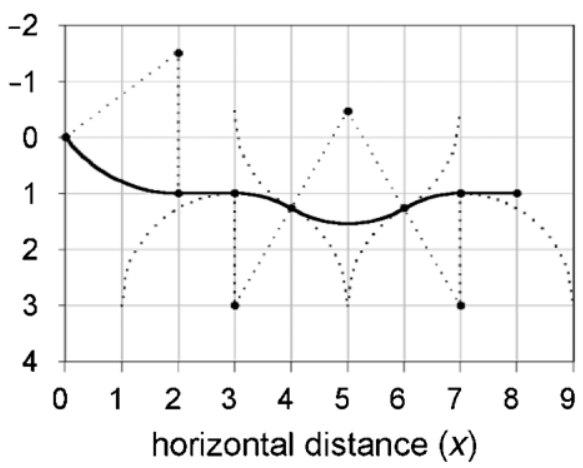

C speed Question (brief version)

Regardless of how quickly either marble reaches any particular point, which marble, if any, is rolling faster at the instant it reaches the vertical line?

* Marble A

* Marble B

* Equal

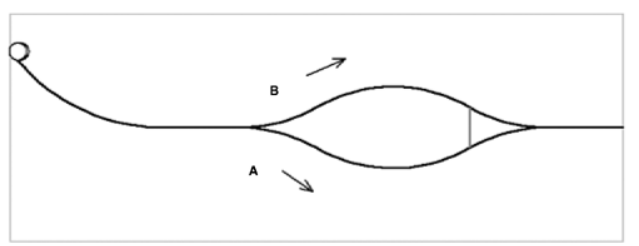

Answer Speed is faster at "uphill point" because its elevation is less (i.e., greater net drop)

Results Most predicted that "downhill point" is faster

Figure 3. The speed and race questions. (A) The hill ramp and dip ramp. These shapes are mathematically specified in Appendix A. (B) The race question. Participants observed a three-dimensional model. (C) The speed question. Participants observed a three-dimensional model.

slope illustrates that the slope-speed belief is a part of their explicit knowledge. It remains unknown, however, whether people also consciously believe that steep "downhill points" are faster than gradual "downhill points"- the second instantiation of the slope-speed belief. Experiment 1 addresses this question.

Experiments 2 and 3 examine whether the slope-speed belief is part of people's implicit knowledge. Both experiments gauge the perceptual realism of computer animations depicting marbles rolling along nonlinear inclines at speeds that conform to either the slope-speed belief or Newtonian theory. If the "slope-speed animations" appear realistic, it would suggest that the slope-speed belief is held implicitly, outside people's awareness. The implications of this finding, as well as a potential origin of the slope-speed belief, are described in the General Discussion.

\section{EXPERIMENT 1}

This experiment required participants to make predictions about incline speed on the basis of a diagram, and the task was designed to rely on their explicit knowledge about motion. Participants estimated the finish speed for two different ramps, as detailed in Figure 4A. Although the precise finish speeds cannot be determined without additional information, the ramps' equal vertical drops ensure equal finish speeds (as explained in the introduction). By the slope-speed belief, however, finish speed is greater at the ramp with the steeper finish point - that is, the convex ramp.

Notably, the two ramps differ with respect to both finish slope and the rolling distance between Points P and Q, but this unavoidable confound worked against the slopespeed belief. That is, the critical section adjoining Points $P$ and $\mathrm{Q}$ on the convex ramp is shorter than the corresponding $\mathrm{P}-\mathrm{Q}$ section along the linear ramp, and the shorter distance allows less "opportunity to gain speed." This is a reasonable (but erroneous) belief that was adopted by some of the participants, thereby reducing the observed support for the slope-speed belief.

\section{Method}

Participants. Fifty undergraduates at the University of South Florida participated in return for course credit. The sample included 41 women and 9 men. 
A
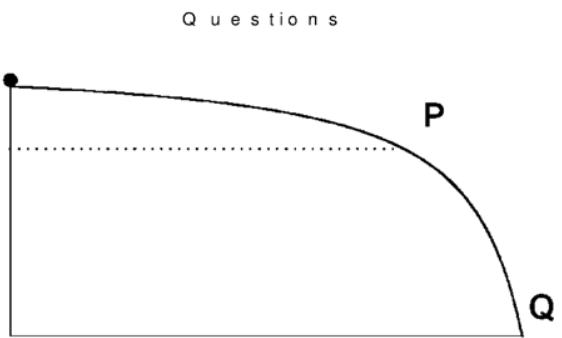

The marble is released at the start.

At Point $\mathrm{P}$, its speed equals 10 .

Estimate the marble's speed at Point Q.

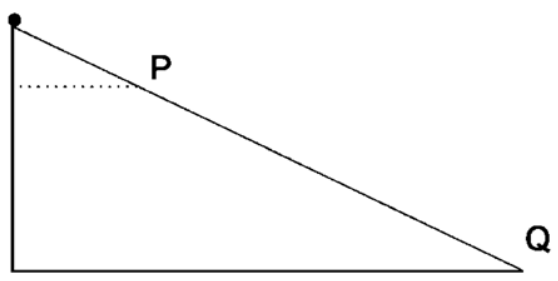

The marble is released at the start.

At Point $\mathrm{P}$, its speed equals 10 .

Estimate the marble's speed at Point Q.

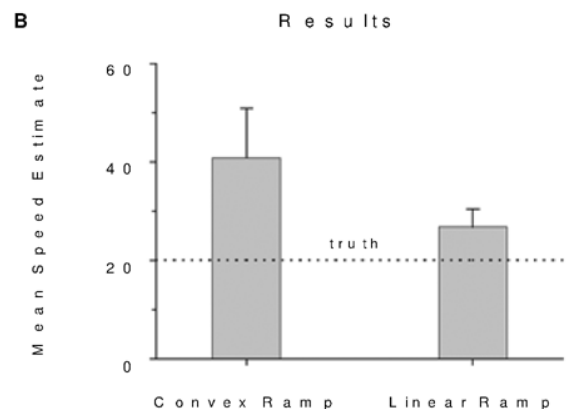

Figure 4. Experiment 1. (A) Questions. Both ramps yield equal finish speed, because speed depends on vertical drop. By the slope-speed belief, finish speed is greater for the convex ramp, because its finish slope is steeper. The speed of 10 at Point $P$ is in arbitrary units. The ramp shapes are specified in Appendix $B$. (B) Results. Positive error bars represent one standard error.

Procedure. Each participant answered the two questions shown in Figure 4A, with the order of these two questions counterbalanced across participants. In the questionnaire, the height of each ramp was $5 \mathrm{~cm}$. The algebraic form of the convex ramp shape is detailed in Appendix B.

\section{Results and Discussion}

The slope-speed belief proved popular, with $60 \%$ of the students predicting incorrectly that the finish speed of the convex ramp would exceed that of the linear ramp. Only $12 \%$ correctly predicted identical speeds for the two ramps, and $28 \%$ predicted greater speed for the linear ramp. (The popularity of the latter response presumably reflects the greater $\mathrm{P}-\mathrm{Q}$ distance along the linear ramp, as described in the introduction to this experiment.) For the slope-speed response, the observed proportion of $60 \%$ was shown by a binomial test to significantly exceed chance $(z=4.00, p<.0001)$. In addition, the mean estimate of finish speed for the convex ramp exceeded that of the linear ramp by more than $50 \%$ (41 vs. 27), as is shown in Figure $4 \mathrm{~B}$. This difference was shown to be statistically significant by the nonparametric Wilcoxon test $(T=270.50$, $p<.001)$.

In summary, most of the participants falsely predicted greater speed at the "steeper point" than at the "gradual point," in spite of an intrinsic confound that worked against the result. This finding is consistent with the erroneous view that an object's incline speed at a "steep downhill point" is necessarily greater than its speed at a "gradual downhill point," consistent with the slope-speed belief. Because this task relied on the participants' explicit knowledge, these results provide further evidence that the slopespeed belief is a part of people's consciously available knowledge. The next two experiments examine whether the slope-speed belief is also a part of their implicit knowledge.

\section{EXPERIMENT 2}

This experiment gauged the perceptual realism of computer animations conforming to the slope-speed belief. Both these "slope-speed animations" and the corresponding Newtonian animations were presented on a computer monitor, with the "marble" rolling down the convex ramp shown in Figure 5A. Participants rated the "naturalness" of each descent.

The task was designed to assess participants' implicit knowledge and to minimize the contribution of explicit knowledge. Specifically, in both the slope-speed animation and the Newtonian animation, the marble continuously gained speed throughout its descent. Furthermore, in both animations, this rate of increase was itself increasing, which is to say that the marble accelerated positively at every point during its descent.

But the two animations differed in degree. As an illustration, Figure 5B indicates the marble's speed at three arbitrary points along the ramp. As shown, the marble's speed during the slope-speed animation initially lags behind its Newtonian counterpart before accelerating dramatically along the final portion. The Newtonian animation did not incorporate friction, and the resulting implications are described in the discussion of this experiment.

\section{Method}

Participants. Twenty-five undergraduates at the University of South Florida participated in return for course credit. This sample included 20 females and 5 males; none had participated in Experiment 1 .

Animations. A Visual Basic computer program paced each animation. The program accommodates nonlinear inclines by varying acceleration with the use of an algorithm written by D.R. and detailed in Appendix C. The "marble" was a red circle with a radius of 
A

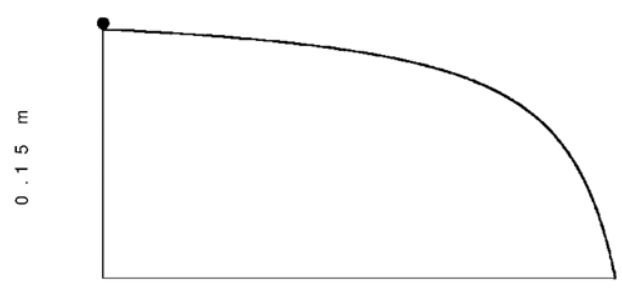

$0.30 \mathrm{~m}$

B

Marblespeed at Various $P$ oints
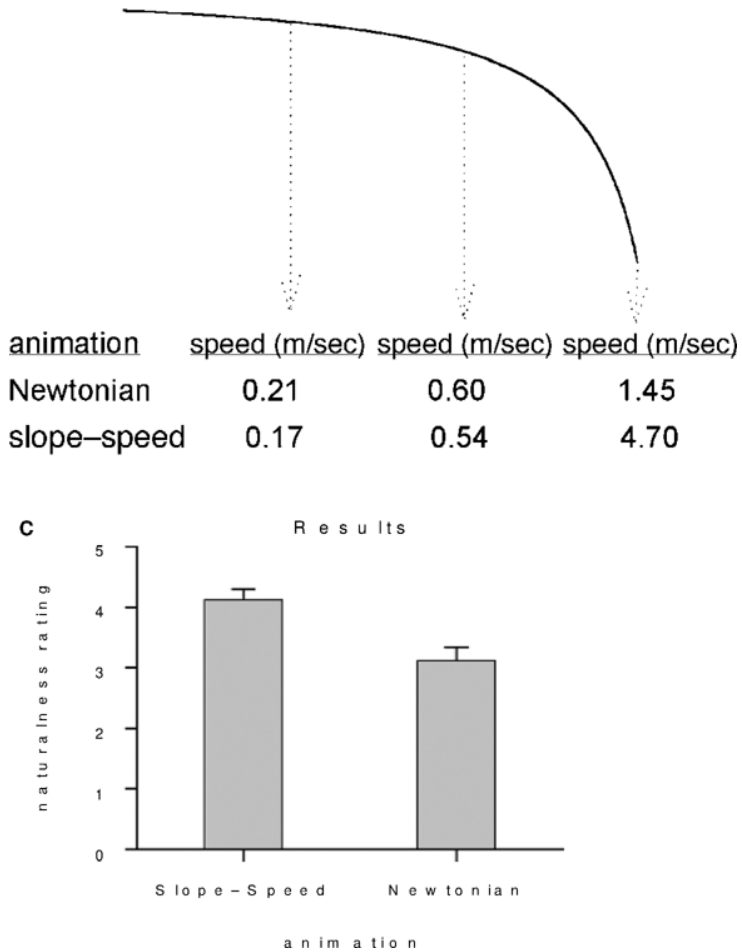

Figure 5. Experiment 2. (A) The ramp. Algebraic specifications are detailed in Appendix B. (B) Speeds at various points. (C) Results. Bar heights represent means, and positive error bars represent $1 S E$.

$2.6 \mathrm{~mm}$, and it descended a blue curve with an approximate thickness of $1 \mathrm{~mm}$. The animation was two-dimensional. The marble included no spokes or other cues about its rotational speed, although participants were told that the red circle represented a rolling marble. At each point along the descent, an imaginary segment adjoining the marble's center and its incline contact point remained perpendicular to the ramp.

For the slope-speed animation, the marble's speed obeyed Equation $\mathrm{C}$, as detailed in Appendix C. This equation was derived so that the marble's speed (in the slope-speed animation) was faster than the Newtonian speed if and only if the slope at that point exceeded the average slope between start and finish. Thus, in the slope-speed animation, incline speed was "too fast" at steeper-thanaverage points and "too slow" at flatter-than-average points. For a linear ramp, consequently, this feature ensures that the slope-speed animation is identical to the Newtonian animation. Equation $\mathrm{C} 1$ intrinsically eliminates most or all of the difference between the duration of the slope-speed descent and the Newtonian descent, depending on the ramp shape. In this study, the Newtonian descent was $14 \%$ quicker than the slope-speed descent $(1,122$ vs. $1,311 \mathrm{msec})$. Thus, the duration is confounded with animation type. However, it is doubtful that the difference in duration is perceptible when the stimuli are presented serially rather than concurrently. The difference was eliminated entirely in Experiment 3 by use of a different ramp shape.

Procedure. Participants were tested individually. The animations were shown on a 17-in. monitor, with the ramp's horizontal distance subtending about $15^{\circ}$ of visual arc. The ramp dimensions are shown in Figure 5A, and the ramp shape is algebraically specified in Appendix B. Each participant viewed the Newtonian animation and the slope-speed animation three times each in an alternating order (i.e., A, B, A, B, A, B). The first to appear was determined randomly. Participants observed the entire sequence before selecting their preference, and the sequence was preceded by the following instruction: "Choose the motion that appears more natural. That is, which choice looks more like it would in the real world, if a marble rolled down a ramp shaped exactly like the one on the screen?" After indicating a preference, each participant provided a rating of each descent's "naturalness" on a 5-point scale.

\section{Results and Discussion}

As can be seen in Figure 5C, the mean naturalness rating of the slope-speed animation $(M=4.12, S E=0.18)$ exceeded that of the Newtonian animation $(M=3.12, S E=$ 0.22 ) by a significant margin $[t(24)=3.54, p<.01]$. In addition, $84 \%$ of the participants rated the slope-speed animation as more natural than the Newtonian animation, with the remaining $16 \%$ choosing the Newtonian animation. A binomial test of proportions revealed that this difference significantly exceeded chance $(z=3.40, p<$ $.001)$. This natural appearance of the slope-speed animation suggests that the slope-speed belief is a part of these participants' implicit knowledge, since the task appears to preclude their reliance on explicit knowledge.

One caveat warrants mention, though, since the perceptual realism of the slope-speed animation may have been influenced by observers' expectations of friction. The magnitude of frictional force is less at points of greater steepness, which is why a toboggan may not begin to slide unless it is moved to a steeper portion of the hill (e.g., Halliday \& Resnick, 1981). Thus, the effect of friction varies with slope in a manner that mirrors one aspect of the slope-speed animation, and this might have contributed to the participants' preference for the slope-speed animation. This rival hypothesis cannot be ruled out in this experiment, although the slope-speed animation differed dramatically from a Newtonian descent under the influence of friction. For example, friction slows an object's speed at every point along the incline, regardless of slope, whereas the slope-speed animation caused the marble to roll at impossibly high speeds near the finish of the convex ramp. This finish speed, as indicated in Figure 5B, was more than three times greater than the finish speed of an optimally quick, frictionless Newtonian descent. Nevertheless, one aim of the next experiment was to explicitly test this rival hypothesis. 


\section{EXPERIMENT 3}

Participants in this study rated the naturalness of animated descents conforming to either the slope-speed belief or Newtonian theory. This experiment differed from Experiment 2 in several respects. First, the animated descents were presented on a large screen rather than on a computer monitor. Second, the animations relied on the dip ramp and hill ramp shown in Figure 3A. Unlike the ramp in Experiment 2, these ramps included both uphill and downhill sections. Third, both a "mild" and a "severe" version of the slope-speed belief were animated, with each participant rating three different animations for each ramp shape: a Newtonian descent, a mild slope-speed de- scent, and a severe slope-speed descent. These modifications were meant to extend the results of Experiment 2 and also to boost ecological validity.

The animations shared several qualitative characteristics. In all three animations, for example, the marble rolled at a constant speed along the horizontal sections of the ramps. Likewise, in each animation, the marble required more time to traverse the "hill" in the hill ramp than to traverse the "dip" in the dip ramp.

Of course, the animations differed in certain respects. The diagram in Figure 6A includes arrows marking the steepest points within the hill or dip, although these arrows were not shown to participants. As indicated, the Newtonian animation produced equal speeds at these two

Marble $S$ peed a t C ritica I Points
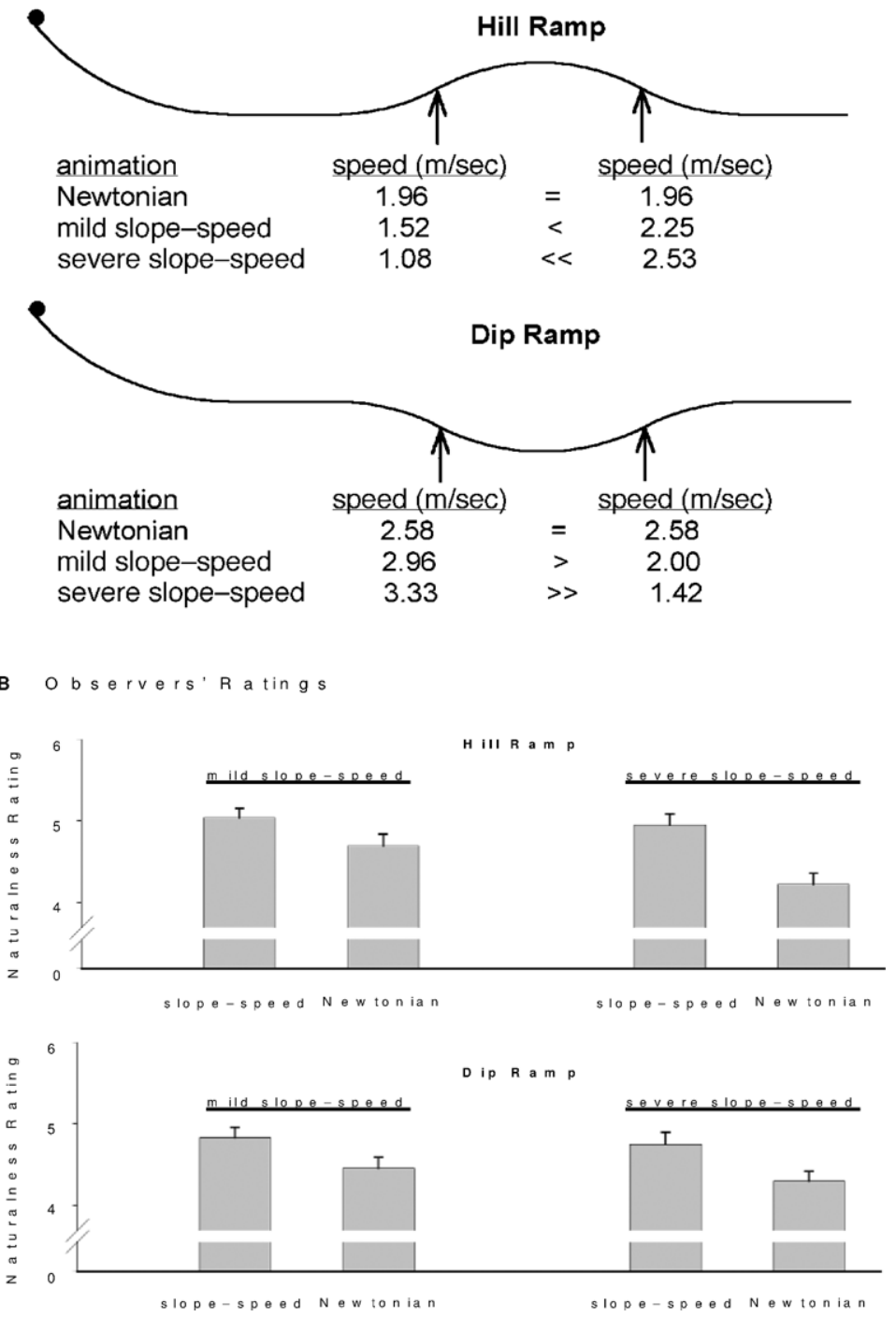

Figure 6. Experiment 3. (A) Illustration of animation differences. The speeds at the two points illustrate one difference between the animations, although these points were not marked in the animation seen by the participants. (B) Results. Bar heights represent means, and positive error bars represent $1 S E$. 
points, because these two points have equal net vertical drops. For the slope-speed animations, however, speed at the "downhill point" was greater than speed at the "uphill point," in keeping with the slope-speed belief.

\section{Method}

Participants. A sample of 120 undergraduates at the University of South Florida participated in return for course credit. The sample included 96 women and 24 men, none of whom had participated in Experiment 1 or 2.

Animations. The animations were projected on a large screen with a resulting horizontal distance of $3 \mathrm{~m}$. The hill ramp and dip ramp shapes are specified in Appendix A and Figure 2A. The animations showed a red "marble" with a radius of $2.6 \mathrm{~cm}$ descending a blue ramp with a thickness of $1.1 \mathrm{~cm}$. The animation technique was otherwise identical to that of Experiment 2.

For both the mild and severe versions of the slope-speed belief, the marble's speed again conformed to Equation C1 in Appendix C. For the mild and severe slope-speed animations and the Newtonian animation, the duration of the hill ramp and dip ramp descents equaled 1,718 and $1507 \mathrm{msec}$, respectively, with an error of $\pm 15 \mathrm{msec}$.

Procedure. Participants were tested in groups, with each participant sitting at a location within $15^{\circ}$ of visual arc from the perpendicular line of sight. Animations were presented in pairs, with each consisting of one slope-speed animation and one Newtonian animation. Each participant observed two pairs of animations for each ramp shape: mild slope-speed versus Newtonian, and severe slopespeed versus Newtonian. In one of these two pairs, the Newtonian animation appeared first. For each pair, participants observed each animation three times in alternation before rating each on a scale from 1 (very unnatural) to 7 (very natural). The critical instruction read as follows: "Give a high rating if you believe that the marble changes speed like it would in the 'real world.' But give a low rating if the marble changes speed in a way that does not appear realistic."

\section{Results and Discussion}

As shown in Figure 6B, observers preferred the slopespeed animation to the Newtonian animation in every condition. Specifically, the mean naturalness ratings of both the mild and severe slope-speed animations significantly exceeded that of the Newtonian animation for both the hill and dip ramps (all $t \mathrm{~s}>2.00$, all $p \mathrm{~s}<.05$ ). This perceptual realism is consistent with the view that the slopespeed belief is part of people's implicit knowledge of incline speed.

Notably, these results cannot be reconciled with a preference for friction-laden descent. For the hill ramp shape, specifically, the addition of friction changes the speed of a Newtonian descent in a manner that is opposite to the deviation given by the slope-speed belief. This is illustrated by the marble's speed at the two critical points on the hill ramp marked by the arrows in Figure 6A. Specifically, in the slope-speed animations, the marble rolled much more slowly at the first indicated point (on the uphill) than at the second point (on the downhill). By contrast, for a Newtonian descent in the presence of friction, the marble would roll faster at the first point than at the second point. This is because friction reduces speed between any two points of equal net vertical drop, which is why a marble eventually stops as it rolls along a horizontal ramp. Therefore, the results of this experiment are counter to the prediction of the rival hypothesis attributing the perceptual realism of the slope-speed animations to friction.

\section{GENERAL DISCUSSION}

Two primary empirical findings are presented. In Experiment 1, participants' estimates of incline speed were greater at the steeper of two "downhill points" despite the equal vertical drops of the two points (Figure 4A). This error is consistent with the slope-speed belief, and, because of the nature of this task, these data suggest that the slope-speed belief is a part of people's explicit knowledge. In Experiments 2 and 3, participants observed computer animations depicting a marble's descent along a nonlinear incline, and they rated animations conforming to the slope-speed belief as more natural than Newtonian animations. This preference was observed for a monotonically declining incline shape (Experiment 2) as well as incline shapes with both uphill and downhill sections (Experiment 3). This perceptual realism of the slope-speed belief suggests that it is held implicitly, outside awareness.

\section{On the Origin of False Beliefs}

Some false beliefs appear to arise from people's poor choice of analogy. For the bomb drop task in Figure 1B, for example, people might simplistically recall that "objects fall straight down when dropped," without accounting for the bomb's speed before it is dropped. Or, they might recall one of countless films showing a stream of bombs falling straight down from a plane without realizing that the camera was inside a second plane flying alongside the first plane. Likewise, the broken pendulum task leads many people to recall having jumped from a swing just before reaching the highest point along the arc, thereby providing horizontal motion during the fall, whereas the suspended bob falls once it reaches the apex. By these explanations, it is not surprising that the animations corresponding to these false beliefs appear unnatural.

Other false beliefs appear to be adaptive and may arise from people's interactions with the environment (e.g., Hecht \& Bertamini, 2000; Hubbard, 1998). As described in the introduction, for example, Hecht and Bertamini found that many people believe that an upwardly thrown object continues to accelerate after it leaves the thrower's hand; Hecht and Bertamini concluded that this belief may facilitate the act of throwing. In effect, the belief serves as a heuristic that is often useful but sometimes misleading. Similarly, there may also be an adaptive role of representational momentum. As described in the introduction, this phenomenon is illustrated by unconscious errors regarding an object's location. These errors are consistent with the effects of physical forces such as gravity and friction, and appear to be intrinsically incorporated in people's implicit knowledge of motion (e.g., Bertamini, 1993; Hubbard, 1995, 1998).

Still others have provided evidence that an explicitly held false belief may be adaptive. As shown by Proffitt and his colleagues, people overestimate a hill's slope when standing at the base of the hill (Bhalla \& Proffitt, 1999; Creem \& Proffitt, 1998; Proffitt, Bhalla, Gossweiler, \& Midgett, 1995). Moreover, these overestimates occur even when people observe the hill from a side view that reveals 
the hill's profile (Proffitt, Creem, \& Zosh, 2001). These authors noted that these overestimates reflect explicit knowledge, and they attribute this overestimation to "response compression." This mechanism effectively magnifies sensitivity to small changes in stimulus magnitude, and, in this case, increases the observer's sensitivity to slight differences in slope. This is useful, because even slight increases in slope induce much greater physiological demands, as evidenced by the sharp decline in people's maximum running speed on a treadmill after a slight increase in the incline slope.

\section{The Origin of the Slope-Speed Belief}

The slope-speed belief may reflect people's experiences with scenarios where slope is, in fact, an excellent predictor of speed. Specifically, if an object is released along an incline, its speed at a subsequent nearby point is strongly predicted by the slope at that point if the incline slope does not change appreciably along the section between these two points. For example, after a skateboarder has rolled several meters along a steep section of road, he/ she is moving quickly. If the skateboarder instead rolls several meters along a gentle slope, he/she is moving slowly. This heuristic fails if the incline includes sharp changes in slope within a narrow region, but such hills are rare.

Slope also provides an excellent predictor of people's incline speed as they walk or run along a hill. When people run at maximum speed, for instance, the slope at a point strongly predicts their maximum speed at that point unless there is a drastic change in slope over a brief distance. Quite simply, people run faster along steep sections and slower along gradual sections. Again, there are exceptions, as is true with any heuristic. For instance, if a runner descends a long steep hill before suddenly beginning a steep uphill, the runner will initially run fast along the uphill before decelerating in the face of gravity. In most scenarios, however, slope roughly predicts incline speed, and these experiences may explain the origin of the slope-speed belief. By this account, the slope-speed belief is a heuristic that facilitates people's negotiation of inclines while impairing their performance on the tasks used in the studies reported here.

\section{REFERENCES}

Ant on, H. (1980). Calculus with analytic geometry (2nd ed.). New York: Wiley.

Ber t a mini, M. (1993). Memory for position and dynamic representations. Memory \& Cognition, 21, 449-457.

Bhal 1 a, M., \& Proffit t, D. R. (1999). Visual-motor recalibration in geographical slant perception. Journal of Experimental Psychology: Human Perception \& Performance, 25, 1076-1096.

Car a mazza, A., McCl oskey, M., \& Green, B. (1981). Naive beliefs in "sophisticated" subjects: Misconceptions about trajectories of objects. Cognition, 9, 117-123.

Cat r a mbone, R., Jones, C. M., Jonides, J., \& Seifer t, C. (1995). Reasoning about curvilinear motion: Using principles or analogy. Memory \& Cognition, 23, 368-373.

Crawf or d, F. S. (1996). Rolling and slipping down Galileo's plane: Rhythm of the spheres. American Journal of Physics, 64, 541-546.

Cr eem, S. H., \& Pr off it t, D. R. (1998). Two memories for geographical slant: Separation and interdependence of action and awareness. Psychonomic Bulletin \& Review, 5, 22-36.

Dr ake, S. (1989). Galileo's gravitational units. The Physics Teacher, 27, 432-436.

Hal 1 iday, D., \& Resn ick, R. (1981). Fundamentals of physics (2nd ed.). New York: Wiley.

Hech t, H., \& Ber t a min i, M. (2000). Understanding projectile acceleration. Journal of Experimental Psychology: Human Perception \& Performance, 26, 730-746.

Hubbar d, T. L. (1995). Environmental invariants in the representation of motion: Implied dynamics and representational momentum, gravity, friction, and centripetal force. Psychonomic Bulletin \& Review, 2, 322-338.

Hubbard, T. L. (1998). Representational momentum and other displacements in memory as evidence for nonconscious knowledge of physical principles. In S. R. Hameroff, A. W. Kaszniak, \& A. C. Scott (Eds.), Toward a science of consciousness II: The second Tucson discussions and debates (pp. 505-512). Cambridge, MA: MIT Press.

Ka iser, M. K., Jonides, J., \& Al exan der , J. (1986). Intuitive reasoning about abstract and familiar physics problems. Memory \& Cognition, 14, 308-312.

Ka iser, M. K., McCl oskey, M., \& Proffit t, D. R. (1986). Development of intuitive theories of motion: Curvilinear motion in the absence of external forces. Developmental Psychology, 22, 67-71.

Ka iser, M. K., Pr off it t, D. R., \& An der son, K. (1985). Judgments of natural and anomalous trajectories in the presence and absence of motion. Journal of Experimental Psychology: Learning, Memory, \& Cognition, 11, 795-803.

Ka iser, M. K., Pr off it t, D. R., Whel an, S. M., \& Hech t, H. (1992). Influence of animation on dynamical judgments. Journal of Experimental Psychology: Human Perception \& Performance, 18, 669-689.

Kozhevnikov, M., \& Hegart y, M. (2001). Impetus beliefs as default heuristics between explicit and implicit knowledge about motion. Psychonomic Bulletin \& Review, 8, 439-453.

$\mathrm{McCl}$ oskey, M., Car amazza, A., \& Green, B. (1980). Curvilinear motion in the absence of external forces: Naive beliefs about the motion of objects. Science, 210, 1139-1141.

McCl oskey, M., \& Koh1, D. (1983). Naive physics: The curvilinear impetus principle and its role in interactions with moving objects. Journal of Experimental Psychology: Learning, Memory, \& Cognition, 9, 146-156.

$\mathrm{McCl}$ oskey, M., Wash bur n, A., \& Fel ch, L. (1983). Intuitive physics: The straight-down belief and its origin. Journal of Experimental Psychology: Learning, Memory, \& Cognition, 9, 636-649.

Proffit t, D. R., Bhal la, M., Gossweil er, R., \& Midget t, J. (1995). Perceiving geographical slant. Psychonomic Bulletin \& Review, 2, 409-428.

Pr off it t, D. R., Creem, S. H., \& Zosh, W. D. (2001). Seeing mountains in mole hills: Geographical-slant perception. Psychological Science, 12, 418-423.

Rohr er, D. (1994). More thought provokers. Berkeley, CA: Key Curriculum Press.

Rohr er, D. (2002). Misconceptions about incline speed for nonlinear slopes. Journal of Experimental Psychology: Human Perception \& Performance, 28, 963-973. 


\section{APPENDIX A}

\section{Specifications for Dip Ramp and Hill Ramp}

The precise specifications of the dip and hill ramps are illustrated by the Cartesian plots in Figure 3A. As shown, each ramp spans a net vertical drop of 1 and a horizontal distance of 8 , yielding an average slope of 1/8. Each unit corresponds to $10 \mathrm{~cm}$ for the apparatus in Rohrer (2002) and to $37.5 \mathrm{~cm}$ in Experiment 3 .

Each ramp is composed of two horizontal line segments and four arcs. Each of these six sections spans exactly one or two horizontal units of the grid. With respect to this grid in Figure $3 \mathrm{~A}$, the six sections are defined as follows:

(1) For $x=[0,2]$, a $53.13^{\circ}$ arc with radius 2.5 and center $(2,-1.5)$

(2) For $x=[2,3]$ and $x=[7,8]$, a horizontal line segment defined by $y=1$

(3) For $x=[3,4]$, a $30^{\circ}$ arc with radius 2 and center at $(3,-1)$ [hill] or $(3,3)$ [dip]

(4) For $x=[4,6]$, a $60^{\circ}$ arc with radius 2 and center at $(5,2.46)$ [hill] or $(5,-0.46)$ [dip]

(5) For $x=[6,7]$, a $30^{\circ}$ arc with radius 2 and center $(7,-1)$ [hill] or $(7,3)$ [dip]

For the speed question in Figure 3C, both comparison points have an $x$-coordinate of 6 and lie at the point of inflection (i.e., where concavity equals zero). Likewise, for the diagram in Figure $6 \mathrm{~A}$, the indicated points have $x$-coordinates of 4 and 6 , respectively, and lie at points of inflection.

Each ramp shape is "everywhere differentiable." That is, at any point conjoining two adjacent sections (e.g., an arc and a line), the slope of both functions is the same at the conjoining point. This eliminates cusps and therefore ensures a smooth roll.

\section{APPENDIX B \\ Convex Ramp Specifications}

The convex shape is hyperbolic. The following derivations yield equations for vertical drop $(y)$ and slope $\left(y^{\prime}\right)$ as a function of horizontal distance $(x)$.

\section{Basic Specifications}

With respect to a Cartesian plane with the positive $y$-axis directed downward, the ramp begins at $(0,0)$ so that $x$ denotes the horizontal distance and $y$ denotes the vertical drop of each point along the ramp. The ramp has a horizontal run of $r$ and a total vertical drop of $d$, so that it ends at $(r, d)$ with average slope $d / r$.

\section{The General Form}

The ramp shape is given by

$$
y=\left(1 / d-s r / d^{2}+s r^{2} / d^{2} x\right)^{-1} .
$$

Substitution reveals that the starting point $(0,0)$ and the finish point $(r, d)$ satisfy Equation B1. The differentiation of Equation B1 yields the slope,

$$
\begin{aligned}
& y^{\prime}=-\left(1 / d-s r / d^{2}+s r^{2} / d^{2} x\right)^{-2}\left(-s r^{2} / d^{2} x^{2}\right) \\
& =s[r s / d-(x / d)(s-d / r)]^{-2} .
\end{aligned}
$$

If $x$ is replaced by its value at the finish point, $r$ (the ramp's run or horizontaldistance), Equation B2 reveals that the slope $\left(y^{\prime}\right)$ at the finish equals $s$.

\section{Ramp with Average Slope of 1/2}

If average slope equals $1 / 2$, then $d / r$ equals $1 / 2$ as well. By substituting $1 / 2$ for $d / r$ in Equation $\mathrm{B} 1$, the vertical drop for a ramp with an average slope of $1 / 2$ is given by

$$
y=(1 / d-2 s / d+4 s / x)^{-1} .
$$

Likewise, substitution of $1 / 2$ for $d / r$ in Equation $\mathrm{B} 2$ reveals the slope,

$$
y^{\prime}=s[2 s-(x / d)(s-1 / 2)]^{-2} \text {. }
$$




\title{
APPENDIX B (Continued)
}

Ramp With Average Slope of 1/2 and Finish Slope of 5 (Experiments 1 and 2)

If finish slope ( $s$ ) equals 5, Equations B3 and B4 simplify further, so that

$$
y=(20 / x-9 / d)^{-1}
$$

and

$$
y^{\prime}=20(20-9 x / d)^{-2} .
$$

The rearrangement of Equation B5 yields an expression for $x$ in terms of $y$ :

$$
x=20 y d /(9 y+d) .
$$

Finally, the expression for $y^{\prime}$ (slope) in terms of the variable $y$ rather than $x$, which is the differential equation, is obtained by replacing $x$ in Equation B6 with the right-hand expression in Equation B7. Subsequent algebraic simplification reveals that

$$
y^{\prime}=\left[20^{1 / 2}+\left(9 * 20^{1 / 2} / d\right) y\right]^{2} \approx[4.472+(40.249 / d) y]^{2} .
$$

\begin{abstract}
APPENDIX C
Animation Technique

The marble's incline speed was determined by numerical integration. The total horizontal distance was divided into 11,600 equal intervals, and the corresponding $(x, y)$ points satisfied either the convex ramp equation (Appendix B) or the dip and hill ramp equations (Appendix A). The line segments adjoining these 11,600 points effectively form part of a polygon that approximates a curve with error less than 1 in $10^{4}$. The "marble" moved along each linear descent at a constant rate, with the duration determined by the following algorithm.

(1) For any line segment, let $\left(x_{1}, y_{1}\right)$ and $\left(x_{2}, y_{2}\right)$ equal the start and end point, respectively.

(2) Consequently, $\Delta y=y_{2}-y_{1}$ and $\Delta x=x_{2}-x_{1}$.

(3) By the Pythagorean theorem, the segment's length $(L)$ is given by $L=\left[(\Delta x)^{2}+(\Delta y)^{2}\right]^{1 / 2}$.

(4) The line segment has an angle of incline $(\theta)$ that is given by $\theta=\arctan (\Delta y / \Delta x)$.

(5) Let $v_{1}$ and $v_{2}$ denote the start speed and end speed of the segment, respectively.

(6) Note that $v_{\mathrm{i}}=\left(k g y_{\mathrm{i}}\right)^{1 / 2}$, as described in the introduction (Equation 1).

(7) Along each linear segment, the average speed ( $\left.v_{\text {avg }}\right)$ equals the mean of $v_{1}$ and $v_{2}$.

(8) The descent duration $(t)$ therefore equals $L / v_{\text {avg }}$ (because distance $=$ rate $*$ time).

For the slope-speed animation, speed at each point was given by the equation,

$$
v_{s-s}=v_{N}\left[b\left(y^{\prime} / s_{\mathrm{avg}}\right)+(1-b)\right],
$$

where $v_{N}$ denotes the Newtonian speed given by the above algorithm, $y^{\prime}$ equals point slope (or the first derivative), $s_{\text {avg }}$ equals the ramp's average slope (net drop/run), and $b$ denotes the severity of the slope-speed belief (0.25 in Experiment 2, and 0.04 or 0.08 in Experiment 3). Notably, Equation $\mathrm{C} 1$ ensures that incline speed exceeds the Newtonian speed if and only if the slope exceeds the average slope. Thus, for linear inclines, slope-speed descent is Newtonian.
\end{abstract}

(Manuscript received March 2, 2002;

revision accepted for publication March 31, 2003.) 